natural processes. We can also confirm that water limits have been reached or breached in many major river basins across the world, and the consequences are already manifest. For example, there is little or no additional streamflow or groundwater for further development remaining in the Murray-Darling River in Australia, the Yellow River in China, the Indus in Pakistan and India, the Amu and Syr Darya in central Asia, the Nile River, and the Colorado River in the United States and Mexico. All of these are important food-producing areas. These basins suffer from excessive pollution, river desiccation, competition for supplies and ecosystem degradation. The drying of the Aral Sea is one of the most infamous examples of ecosystem damage caused by breaching the limits of freshwater withdrawals. Freshwater biodiversity has plummeted as a result of the massive hydraulic construction era beginning in the 1960s. The main driver has been agricultural water use to meet the rising food demands of a growing population.

Johan Rockström and colleagues are suggesting that consumption of 'blue water' sources - evaporation and transpiration from rivers, lakes, groundwater reservoirs and irrigation - should not exceed 4,000 cubic kilometres per year. At present, blue water consumption is estimated at 2,600 cubic kilometres per year. The first thing to say is that the 4,000 figure is based on an analysis of a relatively small number of studies on the global supply and demand of water. When extrapolated (beyond the intentions of the original studies), they lead to a range of 4,000 to 6,000 cubic kilometres. If anything, this 4,000-cubickilometre value may well be too high.

Water for agriculture is one of the forces pushing us beyond our boundary limits. In many areas, dense concentrations of people living on arable land are using water at a rate that has exhausted supplies. In other parts of the world, there is ample water but its use is limited because the land or climate is not suitable for agriculture. In yet other places, such as sub-Saharan Africa, more water could be withdrawn, but expansion in water use is limited by financial and institutional capacity. These variations were not taken into account in the setting of the water boundary.

Another factor not taken into account is the widespread and erroneous assumption that useable water in nature can be readily accessed. In their quest for water and food security, many governments have devised grandiose plans to move massive volumes of water from water-rich to water-poor river basins. Examples include the Interlinking of Rivers Project in India and the South to North Water Diversion Project in China. The ecological consequences of these interbasin water transfers remain unclear, but they are likely to be immense.

Essentially, the concept of a global limit overlooks the importance of local conditions and the role of management in magnifying or ameliorating problems. For this reason, the water boundary suggested by Röckstrom and colleagues may be too high. That said, the planetary boundaries concept and its first estimate of numeric values give us an important warning call that must be heeded. Rather than get bogged down in detailed arguments about the weaknesses of the approach or the methods of analysis, we now have a tool we can use to help us think more deeply - and urgently - about planetary limits and the critical actions we have to take.

Published online: 23 September 2009

doi:10.1038/climate.2009.97

David Molden is the deputy director general for research at the International Water Management Institute, based near Colombo, Sri Lanka.

e-mail:d.molden@cgiar.org

\title{
Consider all consequences
}

\section{PETER BREWER}

\section{Ocean acidification has impacts other than simple changes in $\mathrm{pH}$, and these may need boundaries too.}

$\mathrm{n}$ their definition of planetary boundaries that humans should not transgress for fear of "deleterious or even catastrophic consequences for large parts of the world's inhabitants", Rockström et al. (Nature 461, 472-475; 2009) consider ocean acidification as an essential part of the equation. This may be true whether we consider "inhabitants" to be all life or only humans, for the ocean and its resources are deeply embedded in human culture. But the authors' suggested boundary, based on aragonite saturation - a measure of the extent to which seawater is saturated with the carbonate mineral - needs careful examination.

The term 'ocean acidification' has become the recognizable phrase to encompass the ensemble effects of elevated $\mathrm{CO}_{2}$ levels on marine life. Much as climate is understood to mean much more than temperature change, so too ocean acidification means more than simple changes in $\mathrm{pH}$. Other consequences of warming and the great $\mathrm{CO}_{2}$ invasion of the ocean also need consideration as boundaries. All aerobic life in the sea, not just calcareous animals, will be affected to some degree by the 'acidification' challenge as oxygen levels fall and carbon dioxide levels rise.

Aragonite is the most common form of calcium carbonate used by coralline animals and is the basic building block of coral reefs. Thus, it might be reasonable to expect that if we transgress the proposed boundary for ocean acidification, so that waters at increasingly shallow depths become depleted of aragonite, coral reef

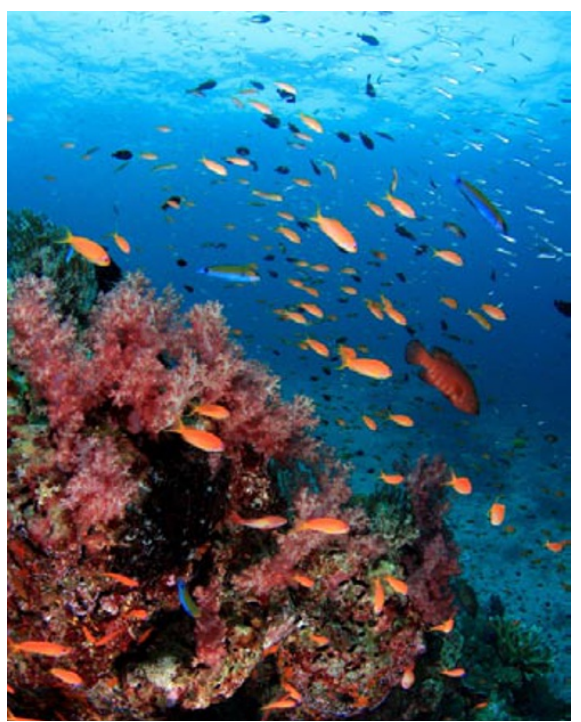


formation will slow substantially. Strong evidence that this can happen has come from many laboratory $\mathrm{CO}_{2}$-manipulation experiments, but there are few comparable field observations of a decline in the growth of large corals at reduced $\mathrm{pH}$.

In fact, many animals form calcareous shells in waters that are well undersaturated with aragonite; the existence of freshwater pearls and deep-sea corals attests to this. These animals have the ability, at a modest physiological cost, to work against the temperature and pressure gradient for dissolution of aragonite.

It is not well-known whether such abilities are latent in reef-forming corals faced with a slow change in $\mathrm{pH}$ over many decades. But the chances are that the species familiar to the reefs we marvel at today will not survive, and we can ill afford to try this global experiment. The limit given by Rockström et al. - an aragonite-saturation state equivalent to at least 80 per cent of the average global pre-industrial level of 3.44 - therefore seems reasonable.

But is it truly useful to create a list of environmental limits without serious plans for how they may be achieved? Without recognition of what would be needed economically and politically to enforce such limits, they may become just another stick to beat citizens with. Disruption of the global nitrogen cycle is one clear example: it is likely that a large fraction of people on Earth would not be alive today without the artificial production of fertilizer. How can such ethical and economic issues be matched with a simple call to set limits? Although peak-oil concerns could be allayed by 'clean' coal technologies, among other solutions, the same cannot be said of phosphate - and food is not optional.

Published online: 23 September 2009

\section{doi:10.1038/climate.2009.98}

Peter Brewer is an ocean chemist and Senior Scientist at the Monterey Bay Aquarium Research Institute in Moss Landing, California.

e-mail:brpe@mbari.org

\section{Rethinking biodiversity}

\section{CRISTIÁN SAMPER}

\section{A boundary that expresses the probability of families of species disappearing over time would better reflect our potential impacts on the future of life on Earth.}

$\mathbf{T}$ he story of life on Earth has unfolded over more than 3 billion years, from the earliest unicellular organisms, through the explosion of diversity in the Cambrian period 530 million years ago, to the amazing diversity of species found on the planet today.
As the paleontological record has improved in recent decades, it has become evident that there have been many periods of mass extinction and that the majority of life on Earth has already become extinct (Extinction: How Life of Earth Nearly Ended 250 Million Years

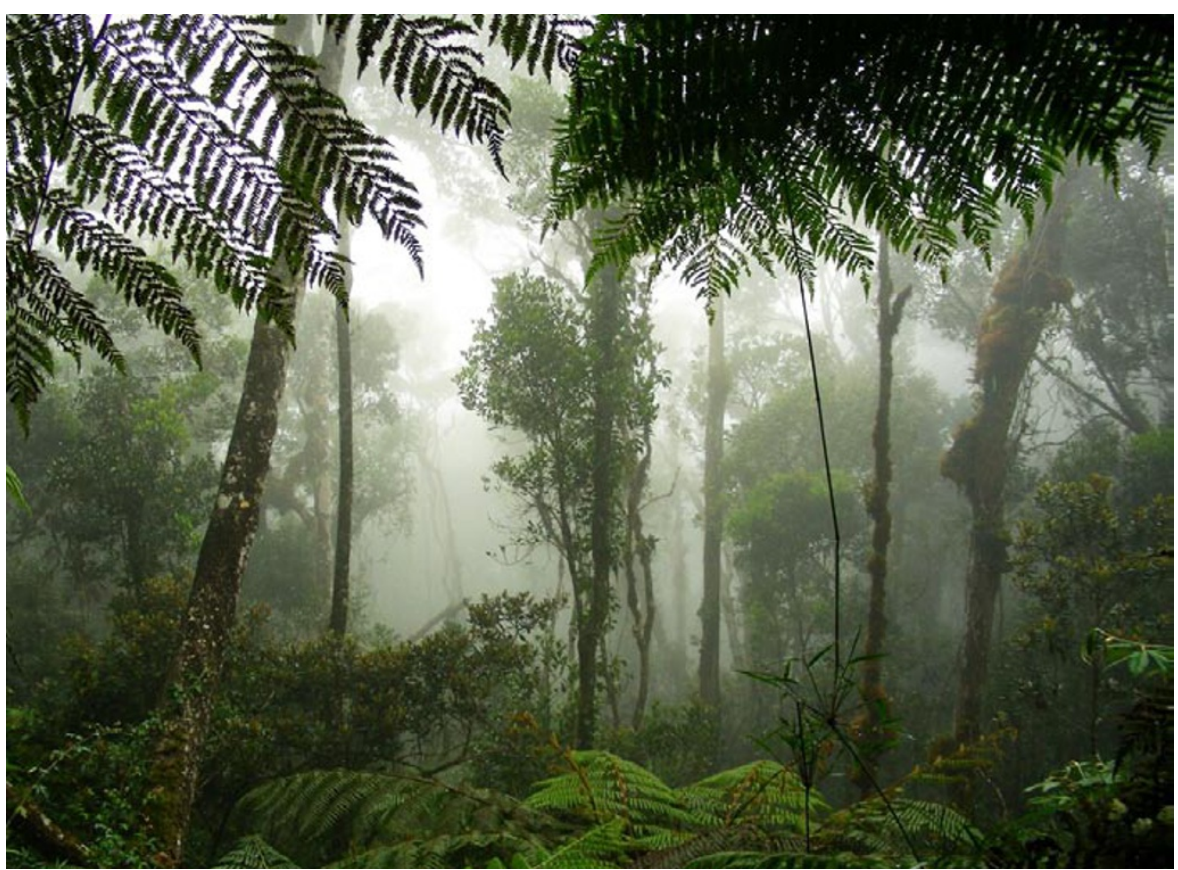

Ago; Princeton University Press, 2006). In comparison, modern humans are relative newcomers to the world stage, dating back just 200,000 years. In that time we have demonstrated a remarkable capacity to transform our environment while needing to adapt to it at the same time.

The planetary boundaries concept presented by Johan Rockström and colleagues (Nature 461, 472-475; 2009) addresses an important question: are there particular thresholds or tipping points beyond which non-linear change would affect the planet?

They believe that one such threshold applies to biological diversity. In their view, extinction of species should not exceed ten species per million per year. If this is exceeded, they argue, we risk irreversible environmental change. Rockström and colleagues conclude that the current rate of extinction -10 to 100 times the average rate - clearly exceeds the proposed boundary.

The first thing to note is that many of the boundaries being proposed are individual physical and chemical variables, which makes them more amenable to measurement over time. The same cannot be said for biodiversity. Interactions among species and ecosystems are extraordinarily complex. Moreover, the 